\title{
Individual and Environmental Risk Factors for COVID- 19 Mortality in Elderly
}

Thomas Bourdrel ( $\nabla$ thomasbourdrel@yahoo.fr)

Laboratoire des Sciences de I'Ingénieur, de I'Informatique et de l'Imagerie

\section{Leo Zabrocki}

Paris School of Economics

\section{Nathalie Compte}

Universitair Ziekenhuis Brussel

\section{Bert Bravenboer}

Universitair Ziekenhuis Brussel

\section{Romain Decours}

Centre Hospitalier Départemental Vendée

\section{Hélène Pelerin}

Centre Hospitalier Départemental Vendée

\section{Laure De decker}

Centre Hospitalier Universitaire de Nantes

\section{Laurence Le Jumeau Kergaradec}

Centre Hospitalier Universitaire de Nantes

\section{Matthieu Lilamand}

Assistance Publique - Hôpitaux de Paris

\section{Claire Roubaud Baudron}

Centre Hospitalier Universitaire de Bordeaux

\section{Bertrand Fougère}

Centre Hospitalier Universitaire de Tours

\section{Rachid Mahmoudi}

Centre Hospitalier Universitaire de Reims

\section{Benoit Schorr}

University Hospital of Strasbourg

\section{Georges Kaltenbach}

University Hospital of Strasbourg

\section{Thomas Vogel}

University Hospital of Strasbourg

\section{Vincent-Henri Puech}

European Centre for Medium-Range Weather Forecasts 
UMR 7357 and CNRS, University of Strasbourg

\section{Marie-Abèle Bind}

Massachusetts General Hospital

\section{Research Article}

Keywords: COVID-19 mortality, Air pollution, Particulate matter, Ultraviolet radiation, Temperature

Posted Date: November 30th, 2021

DOI: https://doi.org/10.21203/rs.3.rs-1033848/v1

License: (c) (i) This work is licensed under a Creative Commons Attribution 4.0 International License. Read Full License 


\section{Individual and Environmental Risk Factors for COVID- 19 Mortality in Elderly}

Thomas Bourdrel ${ }^{* 1}$, Leo Zabrocki*2 ${ }^{* 2}$ Nathalie Compte ${ }^{3}$, Bert Bravenboer ${ }^{3}$, Romain Decours ${ }^{4}$, Hélène Pelerin $^{5}$, Laure De Decker ${ }^{6}$, Laurence Le Jumeau de Kergaradec ${ }^{7}$, Matthieu Lilamand ${ }^{8}$, Claire Roubaud Baudron ${ }^{9}$, Bertrand Fougère ${ }^{10}$, Rachid Mahmoudi ${ }^{11}$, Benoit Schorr ${ }^{12}$,Georges

Kaltenbach $^{12}$, Thomas Vogel ${ }^{12}$,Vincent-Henri Puech ${ }^{13}$, Fréderic Blanc1, Marie-Abèle Bind ${ }^{14}$

(*T.Bourdrel and L.Zabrocki contributed equally to this work')

1ICube Laboratory, UMR 7357 and CNRS, University of Strasbourg, Strasbourg, France.

thomasbourdrel@yahoo.fr frederic.blanc@chru-strasbourg.fr

2 Paris School of Economics - EHESS, Paris ; leo.zabrocki@gmail.com

3 Geriatric department, Universitair Ziekenhuis Brussel,Bruxelles ; nathalie.compte@ uzbrussel.be

bert.bravenboer@uzbrussel.be

4 Service de Gériatrie, Centre hospitalier départemental de Vendée, La Roche Sur Yon ;

romain.decours@ght85.fr;

5 Unité de recherche clinique, Centre hospitalier départemental de Vendée, La Roche Sur Yon

helene.pelerin@ght85.fr

6 Service de Médecine aigüe gériatrique

Centre hospitalier universitaires de Nantes, Nantes ; laure.dedecker@ chu-nantes.fr

7 Service de Gérontologie, Centre hospitalier universitaires de Nantes, Nantes ; laurence.dekergaradec@chu$\underline{\text { nantes.fr }}$

8 Service de Gériatrie

Assistance publique Hôpitaux de Paris (APHP) Nord, matthieu.lilamand@aphp.fr

9 Unité Post Urgences Gériatriques

Centre hospitalier universitaire de Bordeaux, Hôpital Pellegrin, Bordeaux ; claire.roubaud@chu-bordeaux.fr

10 Department of Geriatric

Medicine, Centre hospitalier universitaire de Tours; $\underline{b . f o u g e r e @ g m a i l . c o m ~}$

11 Médecine Interne Gériatrie et Biologie du

Vieillissement, Centre hopitalier universitaire de Reims, Reims ; rmahmoudi@ @ chu-reims.fr

12 Pôle de Gériatrie, La Robertsau Geriatric Hospital, University Hospital of Strasbourg, Strasbourg, France georges.kaltenbach@chru-strasbourg.fr; benoit.schorr@chru-strasbourg.fr thomas.vogel@chru-strasbourg.fr 13 European Centre for Medium-Range

Weather Forecasts. Copernicus

Atmosphere Monitoring Service, London, Royaume-Uni ; Vincent-henri.peuch@ecmwf.int

14 Biostatistics Center, Massachusetts General Hospital, Boston, MA, USA ; ma.bind @ mail.harvard.edu 


\begin{abstract}
Several studies have already explored individual and environmental risk factors for COVID19 morality, however most study populations consisted of the overall population and mainly from China or the US. Our study focused on COVID-19 mortality in the elderly in seven European cities. Long-term exposure to air pollution was estimated through annual pollutant concentrations at the residential address averaged over the last two years of the study period between February and May 2020. We focused on the main outdoor air pollutants $\mathrm{PM}_{10}$, $\mathrm{PM}_{2.5}, \mathrm{NO}_{2}$ and $\mathrm{O}_{3}$. Short-term variations in air pollutants and weather parameters (e.g. temperature, UV, relative humidity) were also examined through a 20-day period before the confirmed PCR diagnostic of COVID-19. Individual risk factors such as smoking status, sex, body mass index (BMI), ischemic heart disease, diabetes, hypertension, chronic renal failure, history of cancer, COPD, and lung fibrosis, were taken into account. We found positive associations for diabetes and COVID-19 mortality (OR 2.2 CI 95\% :1.1, 4.4). Using a multivariate logistic regression model adjusted for all patient characteristics and city, we fail to reject the null hypothesis of no association between COVID-19 mortality and long-term and short-term increase in $\mathrm{PM}_{2.5}, \mathrm{PM}_{10}, \mathrm{NO}_{2}$ and $\mathrm{O}_{3}$. Our study suffers from the fact that patient profiles strongly differ between high-polluted and less-polluted cities. Strong differences in COVID-19 mortalities were observed between cities, which could be due to differences in COVID-19 management and treatment, such as accessibility to reanimation and intensive units between cities. Overall, our study highlights the need to improve estimation of individual exposure to air pollution. Indeed, even with the high-efficiency modelisation systems used in our study, we were unable to estimate the effect of air pollution within each city, because variations in air pollution exposure were too small. Individual markers of air pollution exposure such as recently demonstrated with urinary black carbon or passive individual samplers, would be most suitable for future explorations. Concerning weather parameters, although previous studies concluded that increase in temperature and UV index could decrease COVID-19 morality, our data did not allow us to reject the null hypotheses.
\end{abstract}

Keywords: COVID-19 mortality, Air pollution, Particulate matter, Ultraviolet radiation, Temperature 


\section{Introduction}

Since November 2019, the world is facing a pneumonia epidemic due to a new coronavirus, severe acute respiratory syndrome (SARS)-associated coronavirus 2 (SARS-CoV-2), which leads to a disease named coronavirus disease 2019 (COVID-19)[1]. COVID-19 infections range from completely asymptomatic to severe forms requiring mechanical ventilation. When symptomatic, COVID-19 leads among other symptoms to lower respiratory tract infection, neurological impairment, cutaneous manifestations, or gastro-intestinal issues. Multiple organ failure, myopericarditis, pulmonary embolism, and acute respiratory distress syndrome represent the most serious complications of severe COVID-19. COVID-19 comorbidities are still debated and may include hypertension, obesity, diabetes, cardiovascular disease, chronic obstructive pulmonary disease (COPD), chronic kidney disease, and malignancy [2-4]. As suggested for other respiratory viruses (e.g., SARS-CoV-1,influenza, respiratory syncytial virus, measles), several studies are supporting the role of environmental exposures, such as short- and long-term air pollution exposures, in COVID-19-related morbidity and mortality. In particular, long-term exposure to air pollution through its impact on the development of cardiovascular and respiratory diseases or diabetes may be an aggravating factor for COVID19 morbidity[5]. Short-term variations in air pollution and weathers parameters such as temperature, relative humidity, boundary layer have been correlated with COVID-19 mortality, as well as with the spread of COVID-19 diseases[6-9].

Outdoor air pollution includes gaseous and particulate constituents. Of interest for COVID19, particulate matter (PM) can be simplified into three classifications according to the size of particles, i.e., coarse particles $\left(\mathrm{PM}_{10}\right.$ : diameter less than $\left.10 \mu \mathrm{m}\right)$, fine particles $\left(\mathrm{PM}_{2.5}\right.$ : diameter less than $2.5 \mu \mathrm{m}$ ), and ultrafine particles $\left(\mathrm{PM}_{0.1}\right.$ : diameter less than $\left.0.1 \mu \mathrm{m}\right)$. Depending on their source, PM can have different compositions. Carbonaceous PM from coal, fuel, or wood combustion have been suggested to be the most harmful and responsible for many chronic diseases, mainly cardiopulmonary diseases and cancer [10-11]. The main outdoor gaseous pollutants include nitrogen dioxide $\left(\mathrm{NO}_{2}\right)$ and ozone $\left(\mathrm{O}_{3}\right) . \mathrm{NO}_{2}$ is a major air pollutant in urban European cities, mainly arising from traffic, in particular from diesel cars [5]. $\mathrm{O}_{3}$ is a secondary gaseous pollutant arising from atmospheric photochemical reactions involving sunlight (ultraviolet radiations) and gaseous precursors such as nitrogen oxide (NOx) or volatile organic compounds. As ozone formation requires atmospheric photooxidation of precursors (such as $\mathrm{NO}_{2}$ ), its concentration also depends on meteorological conditions, such as heat and UV. Both $\mathrm{O}_{3}$ and $\mathrm{NO}_{2}$ have been associated with asthma and cardiovascular diseases [12].

Most of the studies that explored individual and environmental risk factors for COVID-19 are US or Chinese surveys and the study population consisted of the overall population. In our study, we investigated the relationship between individual and environmental risk factors and COVID-19 mortality in patients from geriatric units in seven European hospitals, six in France and one in Belgium, see Figure 1 Location of cities. These European cities are interesting to study, because they have different profiles in terms of air pollution and weather parameters. Concerning the association between air pollution and COVID-19 mortality, most 
studies have been conducted at country or regional levels, which can lead to uncertainties, notably with respect to air pollution exposure [13]. In the present study, we used individuallevel data. Our main goal was to explore the influence of long-term exposure to air pollution on COVID-19 mortality. We also explored if short-term variations in air pollutants and weather parameters had an impact on COVID-19 mortality. Concerning individual risk factors, we focused on the main suspected COVID-19 comorbidities such as diabetes and hypertension (HTA).

\section{Methods}

\subsection{Study Population}

All patients were hospitalized in geriatric units of six medium- to large-sized French cities, Strasbourg, Reims, Tours, Nantes, Paris, La Roche sur Yon, and the Belgium capital city, Bruxelles, between February and May 2020. SARS-CoV-2 infection was confirmed by reverse transcriptase polymerase chain reaction (RT-PCR). Because thoracic computerized tomography (CT) scanners were not available for all patients, we did not include thoracic CT examinations for the diagnosis of COVID-19. Smoking status, sex, body mass index (BMI), ischemic heart diseases, diabetes, hypertension, chronic renal failure, history of cancer, chronic obstructive pulmonary disease (COPD), or lung fibrosis were taken into account.

\subsection{Environmental Exposures}

Annual pollutant concentrations averaged over the last two years of the study period, as well as the pollutant concentrations averaged over the 20 day-period before the confirmed PCR diagnostic of COVID-19 were estimated at the residential address. Residential address was translated into Global Positioning System (GPS) coordinates, encoded and transmitted anonymously to the European Centre for Medium Range Weather Forecasts (ECMWF), in charge of the European Commission's Copernicus program implementation. To ensure anonymity, we used only the name of the street and postcode, but not the street number. We obtained written informed consent to participate in the study from the patient's legal guardian. This study was approved by the Ethics Review Committee of the University Hospital of Strasbourg and all methods were carried out in accordance with relevant guidelines and regulations. We exclude patients with missing residential addresses and patients who moved during the last two years of the study period. We focused on the main outdoor air pollutant exposures to $\mathrm{PM}_{10}, \mathrm{PM}_{2.5}, \mathrm{NO}_{2}, \mathrm{O}_{3}$, and sulfur dioxide $\left(\mathrm{SO}_{2}\right)$. We also obtained weather variables such as temperature, relative humidity $(\mathrm{RH})$, ultraviolet radiation (UV), wind speed, boundary layer height, and modelled pollen concentrations for birch, olive tree, grasses, and ragweed, over the 20 day-period before the confirmed PCR diagnostic of COVID-19 from the Copernicus program. Units of measure of environmental factors are detailed in Supplementary Materials Table 1 (Table SM 1: Unit of measure of environmental exposure). 


\subsection{Statistical Analyses}

\subsubsection{Individual and Long-Term Environmental Risk Factors}

First, we estimated the association between long-term exposure to an air pollutant and COVID-19 mortality using a multivariate logistic regression model. We fitted a separate model for each air pollutant. We added to the model patients' characteristics (i.e., sex, age, BMI) and health status (i.e., diabetes, hypertension, COPD, cardiac ischemic disease, renal failure, and cancer). We also adjusted for the patient's city, because it is related to the air pollution exposure and COVID-19 prevalence. Then, we explored the association between long-term exposure to an air pollutant and comorbidities with a multivariate logistic regression model, in which we regressed the occurrence of a comorbidity against an air pollutant concentration and a city indicator. We also investigated the association between patients' characteristics and COVID-19 mortality with a multivariate logistic regression model. The variables added to model were sex, age, BMI, active smoking, diabetes, hypertension, COPD, cardiac ischemic disease, renal failure, and cancer. We again adjusted for the patient's city.

\subsubsection{Short-Term Environmental Risk Factors}

We investigated the short-term influence of air pollutants and weather parameters on COVID-19 mortality with a multivariate logistic regression model. We averaged daily exposure of environmental exposures over the 20 previous before patients undertook a PCR test. We fitted a model for each environmental exposure in which we adjusted for sex, age, BMI, diabetes, hypertension, COPD, cardiac ischemic disease, renal failure, and cancer. We also took into account the patient's city and the month of their PCR test.

\subsubsection{Reproducibility}

All analyses were carried out with the R programming language (version 4.1.0). Although we were not allowed to share the data due to statistical confidentiality, we took great care to provide in the supplementary materials R Markdown documents where coding procedures are fully annotated. https://github.com/lzabrocki/covid_air_pollution

\section{Results}

\subsection{Patients' Characteristics}

We obtained data on 501 patients from seven cities (Supplementary Materials Table 2: Table SM 2 Number of Patients by City). For all patients, we retrieved their residential 
address, sex, age, and other individual risk factors, the date and positivy of their PCR test, and wether they died from COVID-19. The mean age was 83 years (standard deviation=7.5). Female patients represent $49 \%$ of the patients. Several variables, such as active smoking and BMI, presented a non-negligible proportion of missing observations, and to a lesser extent age and cardiac ischemic disease. (Supplementary Materials Table 3: Table SM 3 Proportion of Missing Values by Patients' Characteristics).

The proportion of COVID-19 deaths varied between cities (Figure 2: COVID-19 mortality), ranging from more than $35 \%$ in Strasbourg to less than $20 \%$ in Tours and Reims.

Patient's characteristics were also different between cities (Supplementary Materials Figure SM 1: Patient Characteristics by City), we especially observed different proportions of renal failure, COPD, ischemic cardiopathy, cancer, and diabetes.

\subsection{Long-Term Exposure to Air Pollution}

Figure 3 illustrates the average in air pollutant concentrations between 2017 and 2019 by city. (Figure 3: Average Air Pollutant Concentrations (2017-2019) by City), $\mathrm{PM}_{10}$ levels ranged from 19.6 to $11.3 \mu \mathrm{g} / \mathrm{m}^{3}, \mathrm{PM}_{2.5}$ from 13.5 to $7 \mu \mathrm{g} / \mathrm{m}^{3}$ and $\mathrm{NO}_{2}$ from 27.3 to $3.2 \mu \mathrm{g} / \mathrm{m}^{3}$. Ozone concentrations ranged from 72.2 to $43.9 \mu \mathrm{g} / \mathrm{m}^{3}$..

\subsection{Co-morbidities and COVID-19 Mortality}

Results from the analysis of co-morbidities and COVID-19 mortality are summarized in Table 1 Comorbidities with the Odds of Dying from COVID-19. We found associations for diabetes (OR 2.2, CI 95\%: 1.1, 4.4).

\subsection{Long-Term Air Pollution Exposure and Co-Morbidities}

We also investigated whether air pollution exposures were linked to co-morbidities. We observed some correlations between PM10 and cancer (OR: 1.5, CI 95\%:1.1, 2.1) and between $\mathrm{PM}_{2.5}$ and cancer (OR: 1.5, CI 95\%: 1.1, 2.3) (Figure 4: Long-Term Exposure to Air Pollution and Comorbidities)

\subsection{Long-Term Air Pollution Exposure and COVID-19 Mortality}

Figure 5 and Table 2: Long-term exposure to air pollution and COVID-19 mortality. After adjustment for all patient characteristics and city, our data did not allow us to report an association between long-term exposure to $\mathrm{NO}_{2}, \mathrm{O}_{3}$ and $\mathrm{PM}_{10}$ and the odds of dying from COVID-19. Concerning $\mathrm{PM}_{2.5}$, in the non-adjusted model, we found that a $1 \mu \mathrm{g} / \mathrm{m}^{3}$ increase in long-term exposure to $\mathrm{PM}_{2.5}$ was associated with a positive increase in the odds of dying from COVID-19. However, when adjusted for all patient's characteristics and city, we could not reject the null hypothesis of no association. 
Modelisation of air pollution concentrations did not allow an analysis of the link between air pollution and COVID mortality within cities, because variations of air pollutant concentrations within each city were too small.

\subsection{Short-Term Exposure to Air Pollution and to Meteorological Conditions and COVID-19 Mortality}

Regarding the influence of environmental factors - over a 20 days period before PCR test - on COVID-19 mortality, we could not reject the null hypothesis stating no association between air pollutants, pollens and COVID-19 mortality. However, the study of pollens is limited by the fact that our study period mainly concerns winter months during which no significant pollen concentrations were measured. For a $1 \%$ increase in average humidity, the risk of dying from COVID-19 increased by 1.01 (CI 95\%: 1.00-1.03). Concerning temperature and UV index, our data did not allow us to report an association with the odds of dying from COVID-19 (0.99 CI 95\%: 0.96-1.02 and 0.92 CI 95\% 0.83-1.01, respectively). See Figure 6 and Table 3: Short-Term Environmental Exposure and COVID-19 mortality.

\section{Figures legends:}

Figure 1: Location of cities: map showing the location of the 7 cities.

Figure 2: COVID-19 mortality: proportion of dead patients (\%) in each city

Figure 3: Average Air Pollutant Concentrations (2017-2019) by City: average concentration $\left(\mu \mathrm{g} / \mathrm{m}^{3)}\right.$ for $\mathrm{PM}_{10}, \mathrm{PM}_{2.5}, \mathrm{NO}_{2}, \mathrm{SO}_{2}$ and $\mathrm{O}_{3}$ between 2017 and 2019.

Figure 4: Long-Term Exposure to Air Pollution and Comorbidities: Odds of having COVID-19 comorbidity (i.e cancer, diabetes..) according to average air pollutant exposure between 2017 and 2019.

Figure 5: Long-term exposure to air pollution and COVID-19 mortality: Odds of dying from COVID-19 according to average air pollutants concentration between 2017 and 2019 with adjustment for individual comorbidities (All Patient Characteristics) and for city (City Fixed-Effects).

Figure 6: Short-Term Environmental Exposure and COVID-19 mortality: odds of dying from COVID-19 according to environmental factors changes over a 20 day-period before the confirmed PCR diagnostic of COVID-19. Environmental factors include air pollutants and weather parameters (relative humidity $(\%)$, temperature $\left({ }^{\circ} \mathrm{C}\right)$, pollen $\left(\mathrm{grain} / \mathrm{m}^{3}\right)$, wind speed $(\mathrm{m} / \mathrm{s})$ and ultraviolet radiation (UV index)). 


\section{Discussion}

\subsection{Our Main Findings and Limitations}

\subsubsection{Individual Comorbidities and COVID-19 Mortality}

Concerning individual risk factors, our results indicated that diabetes was associated with a two-fold increased in the odds of dying from COVID-19, which is in line with the results published by previous studies on the main risk factors of COVID-19 mortality [14]. Unlike previous studies, we could not reject the null hypothesis stating no association between COVID-19 mortality and BMI, sex, hypertension, renal failure, and cancer.

\subsubsection{Environmental Risk Factors and COVID-19 Mortality}

\section{Air pollution on COVID-19 mortality:}

Concerning the influence of long-term exposure to air pollution on COVID-19 mortality, in the adjusted models with all patient's characteristics (e.g., diabetes, sex, age), we found that we could not reject the null hypothesis of no association between long-term exposure to $\mathrm{PM}_{2.5}, \mathrm{PM}_{10}, \mathrm{NO}_{2}$, and $\mathrm{O}_{3}$ and the odds of dying from COVID-19. Adjustment with the city indicator indicates that the patient's profiles differ between cities. Indeed, our study revealed differences in COVID-19 mortality between cities that were only partially explained by differences in comorbidities and environmental factors. Other differences between cities such as diet, socio-economic status, or differences in health management, may explain those differences. As an example, accessibility to reanimation and intensive care units may differ between cities, for instance, during this period, intensive care units in Strasbourg and Paris were close to saturation. Even if we do not have the proportion of patients admitted in intensive care units in each city, it is most likely that such differences may have played a role in patient survival. Moreover, our study concerns the first wave of the COVID-19 pandemic, a period during which little was known about COVID-19 management and treatment, which may have led to differences in patient outcomes. For example, some geriatric centers may have introduced corticotherapy and anticoagulants therapies - treatment that has been shown to be effective on survival - whereas others did not in the absence of available scientific evidence at this time of the epidemic.

Concerning the statistical analysis, the strong differences in patient profiles between fairly polluted and less polluted cities did not allow us to analyse the influence of air pollution on COVID mortality using a prospective study design and matching algorithms [15]. Moreover, it would have been helpful to compare the influence of air pollution exposure on COVID-19 mortality within each city, but, even with the high-efficiency modelisation systems used by European Centre for Medium Range Weather Forecasts, we were unable to provide estimates 
of the effect of air pollution within cities, because variations in air pollution exposure were too small.

Our study suffers from several other limitations, such as missing data on patient's characteristics (e.g., BMI, socio-economic status, racial disparities). However, we do believe that, in European cities, such differences are less pronounced and less significant than in the US for instance [16]. Limitation of this study also concerns air pollution exposure that has been estimated through the residential address of the previous two years, which may have led to important misclassification, in particular if patients moved before those two years. In order to ensure patient's anonymity, we did not use the number of the street for the air pollution predictions, which may have resulted in a rough approximation of air pollution exposure. In addition, outdoor air pollutant concentrations may not be representative of individual exposure as elderly patients spend most of their time indoors, particularly patients living in nursing homes. Another limitation is that we did not examine differences between nursing home residents and patients living at home. Nursing home residents may be more vulnerable than independent patients living at home. Regarding air pollution exposure, most previous studies exploring air pollution and COVID-19 mortality have used regional-level data that may lead to inaccuracies in air pollution exposure [17]. As a consequence, our study design with individual-level data based on residential address may be more reliable, but as we discussed before, even individual-level data are imprecise surrogates for personal air pollution exposure. As an example, Travalio et al. used individual-level data from the UK Biobank and found contradictory results depending on the resolution of the modelisation system used for the estimation of air pollution at residential address [18]. Future investigations using individual markers of air pollution may solve this issue. For example, urinary black carbon is accurately correlated with the exposure to air pollution from combustion sources, such as diesel cars, wood, and coal heating [19]. Such individual biomarkers would also be helpful to analyse differences in air pollution exposure within the same city. Indeed, previous studies reported that urinary levels of black carbon strongly differ from one street to another, as a function to the distance from to a main road. Personal passive samplers will also be helpful to improve estimation of individual exposure to air pollutants [20].

Even though there is still no consensus on the role of air pollution in the COVID-19 morbidity and mortality, several studies reported associations between long-term exposure to air pollution and COVID-19 mortality. A Harvard study reported that $1 \mu \mathrm{g} / \mathrm{m} 3$ increase in long-term exposure to $\mathrm{PM}_{2.5}$ was associated with an increase in COVID-19 mortality by $11 \%$ (95\% CI, 6 to 17\%) [21]. Munzel et al. concluded that 15-18\% (95\% confidence interval 7$33 \%$ ) of COVID-19 related-deaths may be attributable to air pollution [22]. Several mechanisms may explain the impact of air pollution on COVID-19 mortality, e.g, it is agreed that long-term exposure to air pollution is associated with an increased risk of chronic diseases, such as ischemic cardiac diseases, lung cancer, COPD, lung fibrosis, diabetes, which are the main suspected co-factors of COVID-19 morbidity and mortality [5]. Furthermore, in-vitro, animals, and human studies demonstrated that even short-term increases in air pollutant exposures lead to a decrease in immune respiratory defense, such as 
macrophage phagocytosis and antimicrobial peptides [5]. Air pollution may also facilitate viral entry due to increased mucosal permeability and activation of protease, which drive the activation of the spike protein [5]. In addition, air pollution may increase renin-angiotensinaldosterone system (RAAS) and Angiotensin II receptor type 1 activity, and thus, may lead to a more severe form of the disease [5]. This brings us to another limitation of our study as we focused on COVID-19 outcomes only in terms of mortality; we recommend that biological parameters such as systemic inflammation, CT scan severity, admission rate in resuscitation units, should be explored in future studies in order to explore the link between air pollution and severity of COVID-19. Air pollution also has indirect harmful effects, for instance, related to lower proportion of UV radiations reaching the atmosphere, air pollution is also associated with reduced levels of vitamin D, which plays an important role in immune response[5].

\section{Weather parameters and COVID-19 mortality:}

Concerning weather parameters, previous studies reported that increase in UV index may lead to decrease COVID-19 mortality, which could be explained by the fact that atmospheric UV may have virucidal activity and are also necessary for vitamin D synthesis [5,23]. In our study, we could not report an association between UV index and COVID-19 mortality. Concerning temperature, previous results from a large study - which explored the OECD countries and the United States - reported that a $1{ }^{\circ} \mathrm{C}$ increase in ambient temperature was associated with a decrease by $6 \%$ of COVID-19 mortality in the next 30 days [9,24]. However we found no associations between temperature and COVID-19 mortality.

We found a positive association between an increase in RH and COVID-19 mortality, however the influence of RH on COVID-19 mortality remains unclear, indeed previous studies reported both positive and negative associations between RH and COVID-19 mortality [25].

In addition to their potential role on COVID-19 mortality, air pollutants and weather variables may be also linked to the spread of the disease, several studies reported that shortterm variations in air pollutants and weather parameters during the fifteen previous days may be associated with an increase of COVID-19 cases [26]. Those effects may be explained by the adverse effect of air pollution on immune response and by the fact that low temperature may reduce the functioning of airways ciliated cells, whereas high temperature and UV radiations may have antiviral activity, but also by the influence of weather conditions on the persistence of viral droplets [5]. Indeed, experimental studies found that SARS-CoV-2 was more stable at low humidity and lower temperatures [5,9]. Relative humidity and temperature may influence hydration and desiccation of viral droplets, and thus may influence the size and contagiousness of droplets [5]. 


\section{Conclusion}

In this multicenter hospital-based study consisting of seven European cities, we found that diabetes status was associated with a 2-fold increase in the odds of dying from COVID-19. Concerning air pollution exposure, we could not reject the null hypothesis of no association between both long-term and short-term exposure and COVID-19 mortality. However, our study suffers from the fact that COVID-19 mortality and patient's characteristics strongly differ between polluted and less polluted cities, and because of the inherent limits of modelisation systems of air pollution exposure, we were not able to precisely estimate the effect of air pollution on COVID-19 mortality within each city. Thus, our study highlights the need for an improved modelisation system at the city level. In the future, individual markers such as urinary black carbon may be helpful to provide better estimation of individual exposure to air pollution. We also suggest that biological outcomes such as systemic inflammation, CT scan severity, admission rate in resuscitation units should be explored in future studies. Concerning meteorological conditions, we could not reject the null hypothesis of no association between UV, temperature and COVID-19 mortality, additional studies are needed to confirm this potential link. We found a positive association between an increase in RH and COVID-19 mortality, however the influence of RH on COVID-19 mortality remains unclear.

Sources of funding: Research reported in this publication was supported by the Office of the Director, National Institutes of Health under Award Number DP5OD021412. The content is solely the responsibility of the authors and does not necessarily represent the official views of the National Institutes of Health.

Conflict of interest statement: The authors declare no competing interests

Code availability : https://github.com/lzabrocki/covid_air_pollution 
Author contributions: T.Bourdrel, F.Blanc and Marie-Abèle Bind contributed to the study conception and design. All autors contributed to data collection. Stastistical analysis were performed by Léo Zabrocki and Marie-Abèle Bind. The first draft of the manuscript was written by T.Bourdrel and all authors commented on previous versions of the manuscript. All authors read and approved the final manuscript.

Ethics approval: This study was approved by the Ethics Review Committee of the University Hospital of Strasbourg (CE-2020-95 / RNI 2020 HUS N ${ }^{\circ}$ 7922). All methods were carried out in accordance with relevant guidelines and regulations

Consent to participate: We obtained written informed consent to participate in the study from the patient's legal guardian.

\section{References}

1.Zhu N, Zhang D, Wang W, Li X, Yang B, Song J, Zhao X, Huang B, Shi W, Lu R, Niu P, Zhan F, Ma X, Wang D, Xu W, Wu G, Gao GF, Tan W; China Novel Coronavirus Investigating and Research Team. A Novel Coronavirus from Patients with Pneumonia in China, 2019. N Engl J Med. 2020 Feb 20;382(8):727-733. doi: 10.1056/NEJMoa2001017

2.Semenzato L, Botton J, Drouin J, Cuenot F, Dray-Spira R, Weill A, Zureik M. Chronic diseases, health conditions and risk of COVID-19-related hospitalization and in-hospital mortality during the first wave of the epidemic in France: a cohort study of 66 million people. Lancet Reg Health Eur. 2021 Sep;8:100158. doi: 10.1016/j.lanepe.2021.100158

3. Cueto-Manzano AM, Espinel-Bermúdez MC, Hernández-González SO, Rojas-Campos E, Nava-Zavala AH, Fuentes-Orozco C, Balderas-Peña LMA, González-Ojeda A, CortesSanabria L, Mireles-Ramírez MA, Ramírez-Márquez JJ, Martínez-Gutiérrez PE, ÁvilaMorán M, De-Dios-Pérez RI, Acosta-Ramírez C, Hernández-García HR. Risk factors for mortality of adult patients with COVID-19 hospitalised in an emerging country: a cohort study. BMJ Open. 2021 Jul 19;11(7):e050321. doi: 10.1136/bmjopen-2021-050321. PMID: 34281931 ; PMCID: PMC8290947.

4. Guan WJ, Liang WH, Zhao Y, Liang HR, Chen ZS, Li YM, Liu XQ, Chen RC, Tang CL, Wang T, Ou CQ, Li L, Chen PY, Sang L, Wang W, Li JF, Li CC, Ou LM, Cheng B, Xiong S, Ni ZY, Xiang J, Hu Y, Liu L, Shan H, Lei CL, Peng YX, Wei L, Liu Y, Hu YH, Peng P, Wang JM, Liu JY, Chen Z, Li G, Zheng ZJ, Qiu SQ, Luo J, Ye CJ, Zhu SY, Cheng LL, Ye F, 
Li SY, Zheng JP, Zhang NF, Zhong NS, He JX; China Medical Treatment Expert Group for COVID-19. Comorbidity and its impact on 1590 patients with COVID-19 in China: a nationwide analysis. Eur Respir J. 2020 May 14;55(5):2000547. doi: 10.1183/13993003.00547-2020. PMID: 32217650; PMCID: PMC7098485.

5. Bourdrel T, Annesi-Maesano I, Alahmad B, Maesano CN, Bind MA. The impact of outdoor air pollution on COVID-19: a review of evidence from in vitro, animal, and human studies. Eur Respir Rev. 2021 Feb 9;30(159):200242. doi: 10.1183/16000617.0242-2020. PMID: 33568525; PMCID: PMC7879496.

6. Rohrer, M., Flahault, A. \& Stoffel, M. Peaks of Fine Particulate Matter May Modulate the Spreading and Virulence of COVID-19. Earth Syst Environ 4, 789-796 (2020). https://doi.org/10.1007/s41748-020-00184-4

7. Dragone, R.; Licciardi, G.; Grasso, G.; Del Gaudio, C.; Chanussot, J. Analysis of the Chemical and Physical Environmental Aspects that Promoted the Spread of SARS-CoV-2 in the Lombard Area. Int. J. Environ. Res. Public Health 2021, 18, 1226. https://doi.org/10.3390/ijerph18031226

8. Yu Wu, Wenzhan Jing, Jue Liu, Qiuyue Ma, Jie Yuan, Yaping Wang, Min Du, Min Liu,

Effects of temperature and humidity on the daily new cases and new deaths of COVID-19 in 166 countries,

Science of The Total

Environment,Vol729,2020,139051,https://doi.org/10.1016/j.scitotenv.2020.139051

9. Christophi, C.A., Sotos-Prieto, M., Lan, FY. et al. Ambient temperature and subsequent COVID-19 mortality in the OECD countries and individual United States. Sci Rep 11, 8710 (2021). https://doi.org/10.1038/s41598-021-87803-w

10. Bourdrel T, Bind MA, Béjot Y, Morel O, Argacha JF. Cardiovascular effects of air pollution. Arch Cardiovasc Dis. 2017 Nov;110(11):634-642. doi: 10.1016/j.acvd.2017.05.003.

11. Schraufnagel DE, Balmes JR, Cowl CT, De Matteis S, Jung SH, Mortimer K, PerezPadilla R, Rice MB, Riojas-Rodriguez H, Sood A, Thurston GD, To T, Vanker A, Wuebbles DJ. Air Pollution and Noncommunicable Diseases: A Review by the Forum of International Respiratory Societies' Environmental Committee, Part 1: The Damaging Effects of Air Pollution. Chest. 2019 Feb;155(2):409-416. doi: 10.1016/j.chest.2018.10.042

12. Atkinson, Richard. W. ; Butland, Barbara. K. ${ }^{\mathrm{a}}$; Anderson, H. Ross. ${ }^{\mathrm{a}, \mathrm{b}}$; Maynard, Robert. L. ${ }^{c}$ Long-term Concentrations of Nitrogen Dioxide and Mortality, Epidemiology: July 2018 Volume 29 - Issue 4 - p 460-472 doi: 10.1097/EDE.0000000000000847 
13. Heederik DJJ, Smit LAM, Vermeulen RCH. Go slow to go fast: a plea for sustained scientific rigour in air pollution research during the COVID-19 pandemic. Eur Respir J. 2020;56(1):2001361. Published 2020 Jul 30. doi:10.1183/13993003.01361-2020

14. Wu, Zh., Tang, Y. \& Cheng, Q. Diabetes increases the mortality of patients with COVID19: a meta-analysis. Acta Diabetol 58, 139-144 (2021). https://doi.org/10.1007/s00592-020$\underline{01546-0}$

15. Imbens, G., \& Rubin, D. (2015). Causal Inference for Statistics, Social, and Biomedical Sciences: An Introduction. Cambridge: Cambridge University Press. doi:10.1017/CBO9781139025751

16. Brandt EB, Beck AF, Mersha TB. Air pollution, racial disparities, and COVID-19 mortality. J Allergy Clin Immunol. 2020;146(1):61-63. doi:10.1016/j.jaci.2020.04.035

17. Berg K, Romer Present P, Richardson K. Long-term air pollution and other risk factors associated with COVID-19 at the census tract level in Colorado. Environ Pollut. 2021 Jun 14;287:117584. doi: 10.1016/j.envpol.2021.117584.

18. Travaglio M, Yu Y, Popovic R, Selley L, Leal NS, Martins LM. Links between air pollution and COVID-19 in England. Environ Pollut. 2021 Jan 1;268(Pt A):115859. doi: 10.1016/j.envpol.2020.115859

19. Saenen ND, Bové H, Steuwe C, Roeffaers MBJ, Provost EB, Lefebvre W, Vanpoucke C, Ameloot M, Nawrot TS. Children's Urinary Environmental Carbon Load. A Novel Marker Reflecting Residential Ambient Air Pollution Exposure? Am J Respir Crit Care Med. 2017 Oct 1;196(7):873-881. doi: 10.1164/rccm.201704-0797OC

20. Mendoza-Sanchez, I., Uwak, I., Myatt, L. et al. Maternal exposure to polycyclic aromatic hydrocarbons in South Texas, evaluation of silicone wristbands as personal passive samplers. J Expo Sci Environ Epidemiol (2021). https://doi.org/10.1038/s41370-021-00348-

21. Wu X, Nethery RC, Sabath MB, Braun D, Dominici F. Air pollution and COVID-19 mortality in the United States: Strengths and limitations of an ecological regression analysis. Sci Adv. 2020 Nov 4;6(45):eabd4049. doi: 10.1126/sciadv.abd4049

22.Andrea Pozzer, Francesca Dominici, Andy Haines, Christian Witt, Thomas Münzel, Jos Lelieveld, Regional and global contributions of air pollution to risk of death from COVID19, Cardiovascular Research, Volume 116, Issue 14, 1 December 2020, Pages 22472253, https://doi.org/10.1093/cvr/cvaa288

23. Gorman S, Weller RB. Investigating the Potential for Ultraviolet Light to Modulate Morbidity and Mortality From COVID-19: A Narrative Review and Update. Front Cardiovasc Med. 2020;7:616527. Published 2020 Dec 23. doi:10.3389/fcvm.2020.616527 
24. Mejdoubi M, Djennaoui M, Kyndt X. Link between COVID-19-related in-hospital mortality in continental France administrative areas and weather: an ecological study. BMJ Open. 2021 Mar 11;11(3):e043269. doi: 10.1136/bmjopen-2020-043269

25. Romero Starke K, Mauer R, Karskens E, Pretzsch A, Reissig D, Nienhaus A, Seidler AL, Seidler A. The Effect of Ambient Environmental Conditions on COVID-19 Mortality: A Systematic Review. Int J Environ Res Public Health. 2021 Jun 21;18(12):6665. doi: 10.3390/ijerph18126665

26. Solimini, A., Filipponi, F., Fegatelli, D.A. et al. A global association between Covid-19 cases and airborne particulate matter at regional level. Sci Rep 11, 6256 (2021). https://doi.org/10.1038/s41598-021-85751-z

\section{Legends :}

$\mathrm{PM}$; particulate matter

$\mathrm{NO}_{2} ;$ Nitrogen dioxide

$\mathrm{O}_{3}$; ozone

$\mathrm{SO}_{2} ;$ sulfur dioxide

HBP ; high blood pressure, hypertension

COVID-19; coronavirus disease 2019

SARS-CoV-2 ; severe acute respiratory syndrome (SARS)-associated coronavirus 2

COPD ; chronic obstructive pulmonary disease

BMI ; body mass index

RT-PCR ; reverse transcriptase polymerase chain reaction

UV ; ultraviolet radiation

$\mathrm{RH}$; relative humidity

$\mathrm{T}^{\circ}$; temperature

OR ; odd ratio

$\mathrm{CI}$; confidence interval

RAAS ; renin-angiotensin-aldosterone system 
Figures

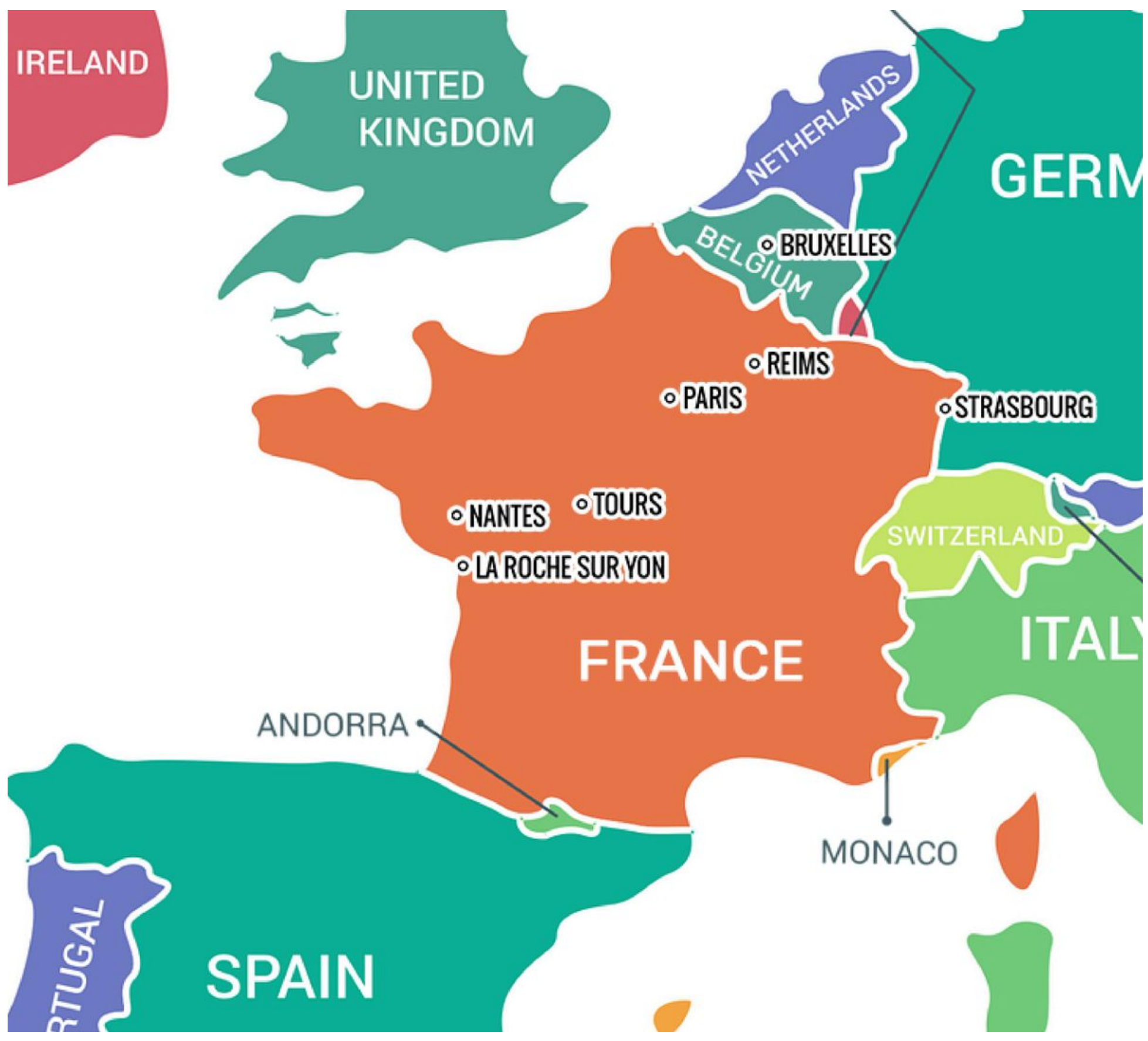

Figure 1

Location of cities: map showing the location of the 7 cities. 


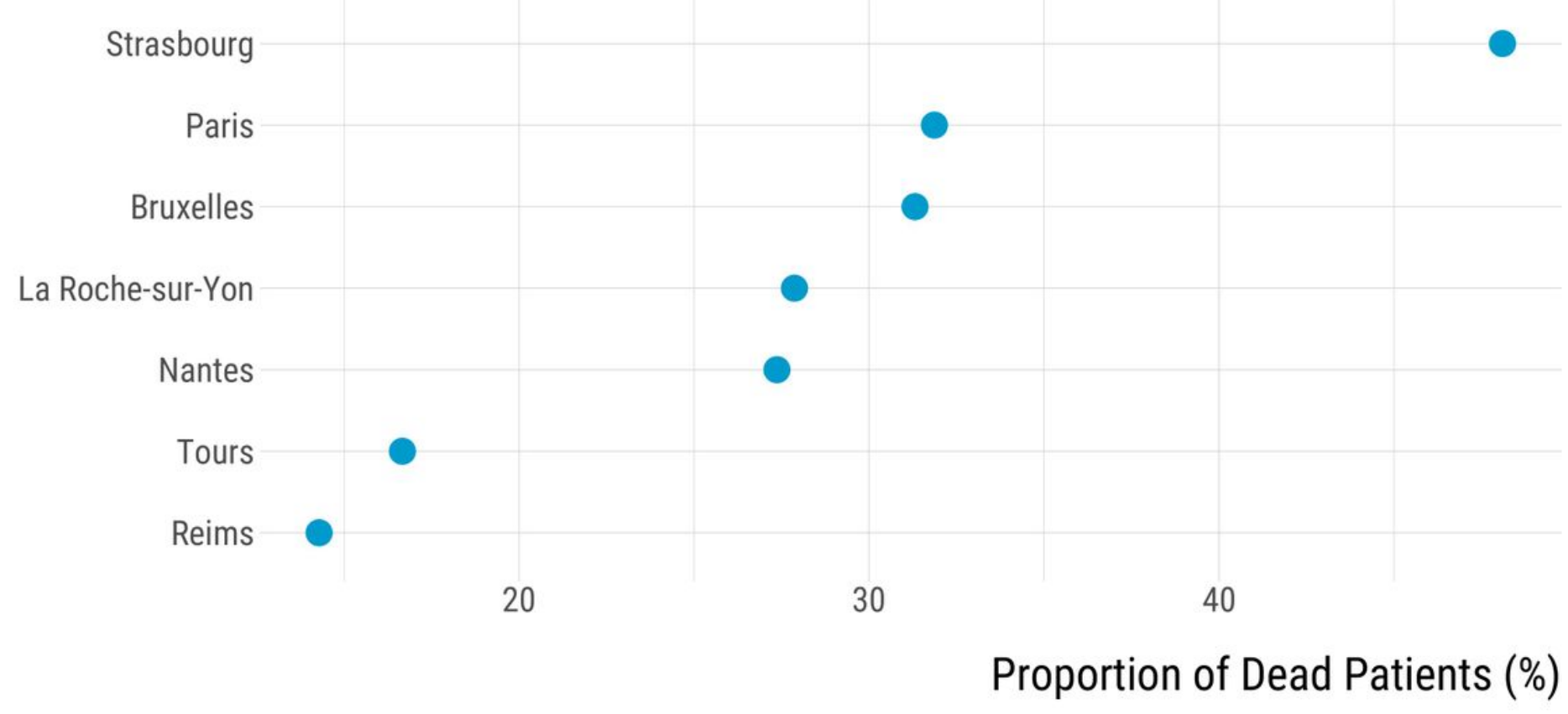

Figure 2

COVID-19 mortality: proportion of dead patients (\%) in each city 


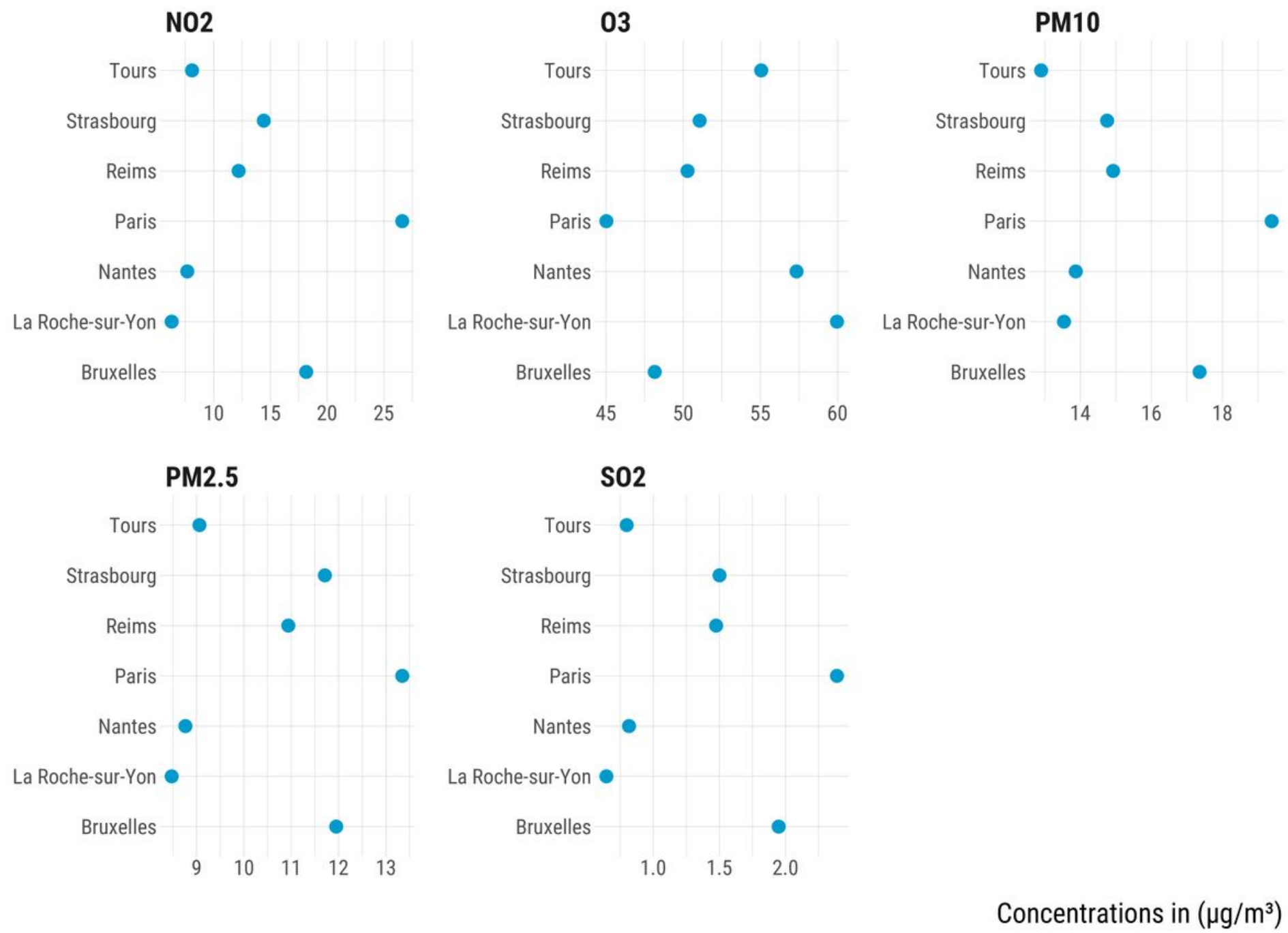

\section{Figure 3}

Average Air Pollutant Concentrations (2017-2019) by City: average concentration $(\mu \mathrm{g} / \mathrm{m} 3)$ for PM10, PM2.5, NO2, SO2 and 03 between 2017 and 2019.

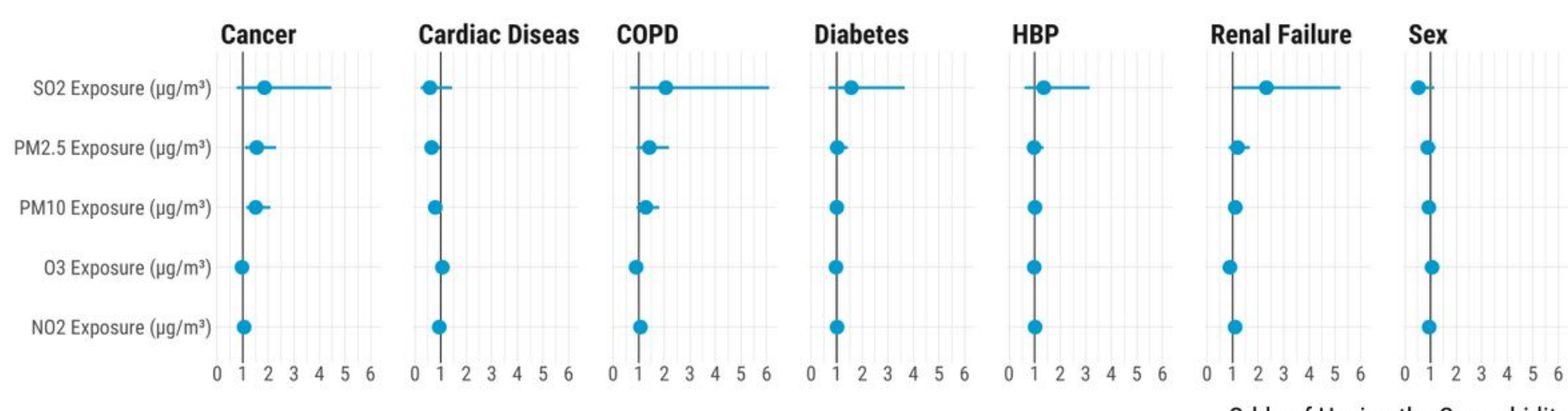

Figure 4 
Long-Term Exposure to Air Pollution and Comorbidities: Odds of having COVID-19 comorbidity (i.e cancer, diabetes..) according to average air pollutant exposure between 2017 and 2019.

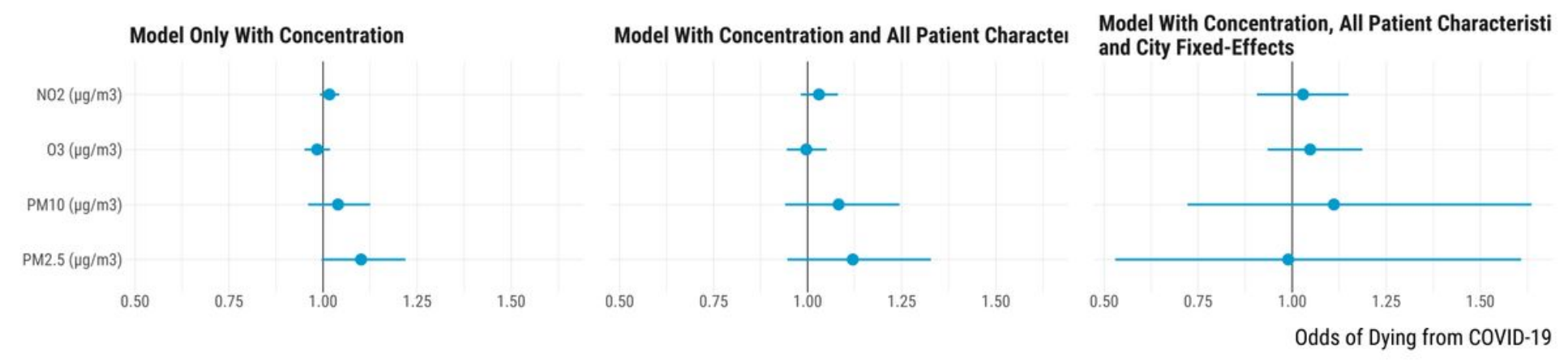

\section{Figure 5}

Long-term exposure to air pollution and COVID-19 mortality: Odds of dying from COVID-19 according to average air pollutants concentration between 2017 and 2019 with adjustment for individual comorbidities (All Patient Characteristics) and for city (City Fixed-Effects).

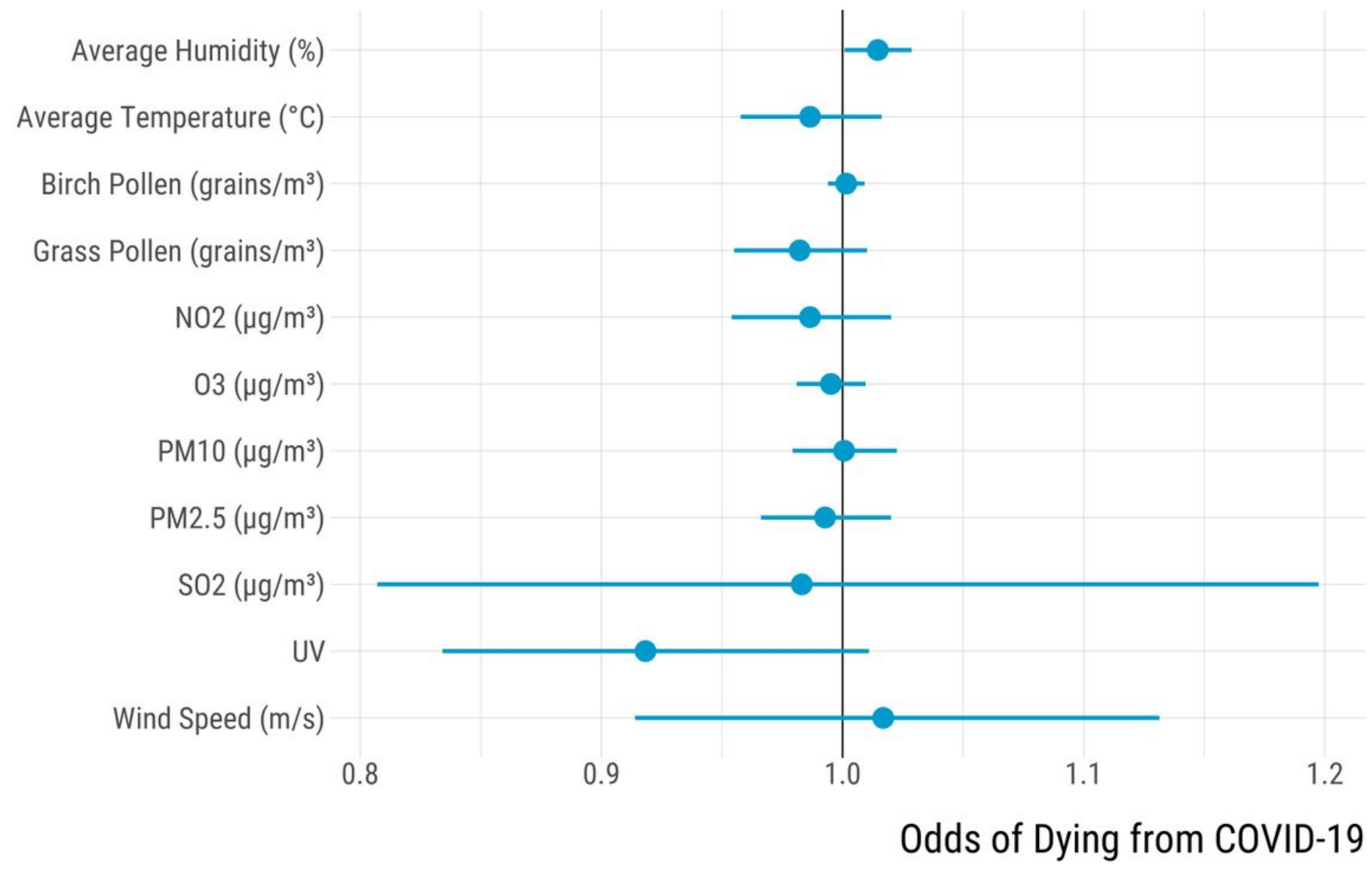

Figure 6 
Short-Term Environmental Exposure and COVID-19 mortality: odds of dying from COVID-19 according to environmental factors changes over a 20 day-period before the confirmed PCR diagnostic of COVID-19. Environmental factors include air pollutants and weather parameters (relative humidity (\%), temperature $\left({ }^{\circ} \mathrm{C}\right)$, pollen (grain/m3 ), wind speed $(\mathrm{m} / \mathrm{s})$ and ultraviolet radiation (UV index)).

\section{Supplementary Files}

This is a list of supplementary files associated with this preprint. Click to download.

- Table1.pdf

- Table2.pdf

- Table3.pdf

- SMFig1PatientCharacteristicsbyCity.pdf

- TableSM1.pdf

- TableSM2.pdf

- TablesM3.pdf 